\title{
Review of: "Mangroves are an overlooked hotspot of insect diversity despite low plant diversity"
}

\author{
Luis Duarte
}

Potential competing interests: The author(s) declared that no potential competing interests exist.

The study compare the tropical arthropod fauna across a freshwater swamp and six different forest types. The authors have pointed out that mangroves is an overlooked hotspot for insect diversity and call attention for even plant diversity is a good predictor of insect diversity, mangroves are an exception. The study brings valuable results that corroborate it in a scientifically innovative way. Graphics are very informative. The organization os ideias and the discussion addressed convince the reader of It and reinforce that mangroves needs to be preserved and better explored about its diversity. 